\title{
CORRÊNCIA DE POTENCIAIS AGENTES CAUSADORES LARVA MIGRANS EM PARQUES E PRAÇAS PÚblicas em APARECIDA DE GoIÂnIA, GoIÁs, Brasil
}

\section{Nathália Miguel Costa Monteiro}

Universidade de Rio Verde, Faculdade de Medicina de Aparecida de Goiânia, Goiás, Brasil. E-mail: natymigmonteiro1@gmail.com

\section{Carolina Araújo Gonçalves}

Universidade de Rio Verde, Faculdade de Medicina de Aparecida de Goiânia, Goiás, Brasil. E-mail: carolaraujog000@gmail.com

\section{Alexandre Abdelaziz Rodrigues}

Universidade de Rio Verde, Faculdade de Medicina de Aparecida de Goiânia, Goiás, Brasil. E-mail: alexandrear94@gmail.com

\section{Rafael Campos Oliveira}

Universidade de Rio Verde, Faculdade de Medicina de Aparecida de Goiânia, Goiás, Brasil. E-mail: rafacampim@gmail.com

\section{Jaqueline Ataíde Silva Lima}

Universidade Federal de Goiás, Instituto de Patologia Tropical e Saúde Pública, Laboratório de Estudos da Relação Parasito-Hospedeiro. E-mail: Jaquelinellima21@gmail.com

\section{Juliana Boaventura Avelar}

Universidade Federal de Goiás, Instituto de Patologia Tropical e Saúde Pública, Laboratório de Estudos da Relação Parasito-Hospedeiro. E-mail: julianabavelar@gmail.com

\author{
Ana Maria de Castro \\ Universidade Federal de Goiás, Instituto de Patologia Tropical e Saúde Pública, Laboratório de \\ Estudos da Relação Parasito-Hospedeiro. E-mail: amaria.ana@gmail.com
}

\section{HÂnstter Hállison Alves Rezende}

Universidade Federal de Goiás, Regional Jataí, Unidade Acadêmica Especial de Ciências da Saúde. E-mail: hanstter@gmail.com

\begin{abstract}
Resumo: O solo é uma importante via de transmissão de agentes etiológicos de zoonoses, dentre eles os parasitos. O objetivo neste estudo foi analisar amostras de solo de praças e/ou parques no município de Aparecida de Goiânia-GO, com o intuito de identificar parasitos responsáveis por causar larva migrans. Para isto, foram avaliadas 30 praças do município, com a coleta de três amostras em cada local. A análise parasitológica foi realizada com a técnica de Willis. Das 30 praças avaliadas, $11(36.7 \%)$ estavam contaminadas com parasitos que podem causar larva migrans. Das 90 amostras, 14 (15\%) foram positivas. A prevalência de ovos de Toxocara sp. foi de $12.2 \%$ e Ancilostomatídeos de $3.3 \%$. O relato da presença destes parasitos em praças públicas fornece informações sobre a contaminação destes locais no município de Aparecida de Goiânia-GO e pode contribuir com o estímulo à implantação de medidas profiláticas que reduzam esses parasitos nas áreas de lazer.
\end{abstract}

Palavras-chave: larva migrans, helmintos, saúde pública. 


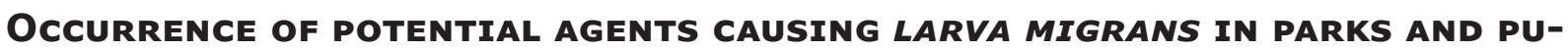 BLIC SQUARES IN APARECIDA DE GOIÂNIA, GOIÁS STATE, BRAZIL.
}

\begin{abstract}
The soil is an important route of transmission of etiological agents that cause zoonoses, among them the parasites. The objective in this research was to analyze soil samples from squares and/ or parks in the city of Aparecida de Goiânia-GO, in order to identify parasites responsible for causing larva migrans. For this, 30 squares in the city were evaluated and tree samples were collected from each one. The parasitological analysis was performed using the Willis's technique. Among the 30 squares evaluated, $11(36.7 \%)$ were contaminated with parasites that can cause larva migrans. Of the 90 samples, $14(15 \%)$ were positive. The prevalence of eggs of Toxocara sp. was $12.2 \%$ and Ancylostomidae of $3.3 \%$. The presence of these parasites in public squares provides information about their contamination in Aparecida de Goiânia-GO and can contribute to stimulate the implantation of prophylactic measures that reduce these parasites in recreational areas.
\end{abstract}

Keywords: larva migrans, helminths, public health.

\section{INTRODUÇÃO}

As parasitoses entéricas são frequentes na população brasileira constituindo um sério problema de saúde pública. Estas doenças geralmente negligenciadas e ocorrem principalmente em regiões economicamente desfavorecidas, com os fatores de risco relacionados diretamente com condições de higiene, educação sanitária e ambiental (Mascarini et al., 2010; Bortolatto et al., 2017).

O solo se torna uma importante via de transmissão para essas zoonoses, pelo fácil acesso de animais, como cães e gatos, aos locais de lazer infantis, o que aumenta exposição da população frequentadora destes locais, sobretudo as crianças (Bortolatto et al., 2017; Júnior et al., 2015).

O quadro clínico de larva migrans, pode ser causado por larvas dos parasitos do gênero Toxocara sp. e por Ancilostomatídeos (Sommerfelt et al., 2006; Rodrigues et al., 2014).

As larvas de Ancilostomatídeos são responsáveis pela síndrome da larva migrans cutânea (LMC), popularmente conhecida como bicho-geográfico (Bowman et al., 2010). O clima quente e o solo úmido sombreado são condições favoráveis ao desenvolvimento embrionário desses parasitos, que podem permanecer no solo por meses (Borges et al., 2013). A LMC ocorre pela migração do parasito pela pele, que gera forte prurido, e leva o paciente muitas vezes a se coçar, provocando escoriações na pele e infecções microbianas secundárias (Farias et al., 2013, Gupta, 2016; Tekely et al., 2013). Como os humanos são os hospedeiros acidentais, as larvas sobrevivem no organismo apenas por duas a quatro semanas, sendo a doença autolimitada em mais de $80 \%$ dos casos (Gupta, 2016). É mais comum o acometimento dos membros inferiores, pés, nádegas e mãos (Tekely et al., 2013; Leite, 2013; Alves \& Proença, 2012).
Já o quadro de larva migrans visceral (LMV) ocorre pela ingestão acidental de ovos embrionados de Toxocara canis Werner, 1782 ou Toxocara cati. Schrank, 1788 parasitos de cães e gatos, respectivamente (Mota et al., 2016; Souza et al., 2011). Ao atingirem o intestino, os ovos eclodem e as larvas migram pela via linfática ou circulação portal para diversos órgãos, como pulmão, fígado, coração e cérebro (Farias et al., 2013; Souza et al., 2011; Brener et al., 2008). Essas larvas também podem migrar através da circulação sanguínea e causar larva migrans ocular (LMO), que é a manifestação menos comum da infecção por Toxocara sp., sendo mais comum em pacientes jovens e ocorrendo geralmente de forma unilateral (Hare \& Franco-Paredes, 2014).

A prevalência de larva migrans em humanos em Goiânia, Goiás foi realizada por meio da avaliação de anticorpos anti-T.canis em amostras de 1.131 pacientes, sendo detectada uma prevalência de 18,9\% (Santos et al., 2009).

Dessa forma, teve-se como objetivo analisar amostras de solo de parques e/ou praças do município de Aparecida de Goiânia, com o intuito de identificar parasitos responsáveis por causar as diversas formas de larva migrans.

\section{MATERIAL e MÉtodos}

Trata-se de um estudo descritivo, realizado no município de Aparecida de Goiânia-GO. É um dos municípios da Região Metropolitana de Goiânia e possui uma população estimada de 532.135 habitantes (IBGE, 2017).

Foram coletadas amostras de 30 praças e/ou parques de 19 bairros do município, no período setembro de 2016 a dezembro de 2016. Em cada local, foram três amostras de cerca de 250 gramas, totalizando 90 amostras. As amostras foram colhidas com auxílio de um coletor descartável em três pontos diferentes, com preferência para os locais que possuíam predominantemente areia 
ou terra, com profundidade de no máximo oito centrímetros.

Cada amostra foi acondicionada em um saco plástico descartável, identificada e mantida no refrigerador até sua análise. O tempo entre a coleta e processamento foi de no máximo três dias. O processamento e a análise das amostras de solo foram realizados no Laboratório de Estudos da Relação Parasito-Hospedeiro do Instituto de Patologia Tropical e Saúde Pública da Universidade Federal de Goiás.

A técnica de Willis (Willis, 1921), foi utilizada para a pesquisa de ovos de parasitos, pois é indicada como técnica padrão ouro para ovos de Ancilostomatídeos (Novaes \& Martins, 2015). Ela fundamenta-se na flutuação espontânea em cloreto de sódio, na qual os ovos aderem à superfície inferior de uma lâmina colocada na parte superior do líquido.

Para cada amostra, foram realizadas três lâminas com adição de lugol. Cada lâmina foi observada ao microscópio óptico nos aumentos de 100X e 400X, os parasitos foram identificados com base nos padrões morfológicos dos ovos, sendo utilizada a ocular micrométrica para a confirmação.

\section{Resultados}

Das 30 praças avaliadas, foram constatadas contaminações com parasitos em 11, perfazendo um percentual de 36,7\%. Das 90 amostras analisadas, 15,5\% (14/90) foram positivas, sendo $12,2 \%$ para ovos de Toxocara sp. e 3,3\% de ovos de Ancilostomatídeos como demonstrado na Tabela 1.

Tab. 1. Distribuição dos parasitos encontrados e sua prevalência nas praças e parques do município de Aparecida de Goiânia, no ano de 2016.

\begin{tabular}{|c|c|c|}
\hline \multirow{2}{*}{ PARASITOS } & \multicolumn{2}{|c|}{ MOSTRAS POSITIVAS } \\
\hline & $\mathbf{N}(90)$ & $\%$ \\
\hline Ovo de Toxocara sp. & 11 & $12,2 \%$ \\
\hline Ovo de Ancilostomatídeos & 3 & $3,3 \%$ \\
\hline Amostra negativa & 76 & $84,5 \%$ \\
\hline
\end{tabular}

\section{DIscussão}

As coletas das amostras ocorreram na primavera entre setembro e dezembro de 2016, sendo uma estação favorável para a evolução dos ovos de Toxocara sp. e Ancilostomatídeos. Portanto, a temperatura era favorável para o desenvolvimento dos parasitos (Carvalho \& Rocha, 2011; Moro et al., 2008).

Oliveira et al. (2007), observaram que 30\% das amostras coletadas do solo das praças em creches municipais de Santa Maria estavam contaminadas com ovos de Ancylostoma sp. e Toxocara sp., semelhante aos $36,7 \%$ de contaminação obtidos em nossa pesquisa.

Bertolatto et al. (2017), verificaram contaminação semelhante, em que $39 \%$ do solo de praças e parques em Caxias do Sul-RS possuíam formas parasitárias.

No estudo realizado por Guimarães et al. (2005) para a presença de Toxocara sp. e Ancylostoma sp. em praças públicas de Lavras- MG, a contaminação foi de $69,6 \%$ das praças, assim como na pesquisa realizada por Souza et al. (2007)em que a positividade para a contaminação por algum desses parasitos foi de $100 \%$ nas praças da Zona Sul do Rio de Janeiro. Esses resultados são muito superiores aos encontrados em nossa pesquisa $(36,7 \%)$, provavelmente devido ao maior acesso dos animais às praças de ambas as localidades.

O resultado encontrado na presente pesquisa para contaminação por Toxocara sp. (33,33\%) é semelhante ao encontrado por Santarém et al.(2012), em que 38,7\% dos lotes analisados mostraram contaminação por ovos de Toxocara sp. em assentamento rural de Mirante do Paranapanema, São Paulo. No estudo feito por Capuano \& Rocha (2005) em praças públicas de Ribeirão Preto, concluiu-se que $20,5 \%$ de 78 praças analisadas estavam contaminadas por ovos de Toxocara sp., semelhante aos $20 \%$ em nossa pesquisa. No estudo realizado por Souza et al.(2007), também foi encontrado maior contaminação por ovos de Toxocara sp. (100\%) do que por Ancilostomatídeos (25\%).

Ribeiro et al. (2013) ao analisar a areia de praças do município de Esteio (RS), verificaram que $88,2 \%$ estavam contaminados com ovos de Ancylostoma sp. e 52,9\% por ovos de Toxocara sp. Brenner et al. (2008) avaliaram 60 praças do Rio de Janeiro e concluiu que em 16 foi verificada a presença de ovos de Ancylostoma sp. e em duas praças a presença de ovos de Toxocara sp.

Este estudo representa o primeiro relato de potenciais agentes causadores larva migrans em Aparecida de Goiânia-GO. Os resultados desta pesquisa comprovaram a presença de potenciais agentes de larva migrans no solo dos locais pesquisados. Dessa forma, são necessárias ações para a conscientização da população e principalmente dos proprietários de animais domésticos, que ao saírem para passear com seus animais de estimação, recolham as fezes que esses deixam durante o passeio. Além disso, a realização da profilaxia parasitária nos animais de companhia, bem como o controle de animais errantes também devem ser observados. É importante também que 
sejam instaladas grades de contenção para evitar o acesso dos animais aos locais de recreação.

\section{REFERÊnCIAS}

Alves, C. \& V. Proença. 2012. Larva migrans cutânea - um caso de apresentação típica no viajante. Rev. Port. Med. Geral. e Fam. 28:136-138.

Borges, A. D., G. M. Tshibangu, C. G. P. Beyrodt \& W. Barrella. 2013. Presença de larva migrans em areas de lazer nas creches, escolas infantis municipais e praças públicas de Salto de Pirapora, SP. Rev Elet. Biol. 6(1):94101.

Bortolatto, J. M., M. M. Sniegovski, T. S. Bernardi, L. B. Crippa \& A. D. Rodrigues. 2017. Prevalence of parasites with zoonotic potential in soil from the main public parks and squares in Caxias do Sul, RS, Brazil. Rev. Patol. Trop. 46(1):85-93.

Bowman, D. D., S. P. Montgomery, A. M. Zajac, M. L. Eberhard ML \& K. R. Kazacos. 2010. Hookworms of dogs and cats as agents of cutaneous larva migrans. Trends Parasitol. 26(4):162-167.

Brener, B., D. P. B. G. Mattos, P. R. Millar, E. K. N. Arashiro, V. Duque-Ferreira \& A. P. Sudré. 2008. Estudo da contaminação de praças públicas de três municípios do Estado do Rio de Janeiro, Brasil, por ovos e larvas de helmintos. Rev. Patol. Trop. 37(3):247-254.

Capuano, D. M. \& G. M. Rocha. 2005. Environmental contamination by Toxocara sp. eggs in Ribeirão Preto, São Paulo State, Brazil. Rev. Inst. Med. Trop. Sao Paulo. 47(4):223-226.

Carvalho, E. A. A. \& R. L. Rocha. 2011. Toxocaríase: larva migrans visceral em crianças e adolescentes. J. Pediatria. 87(2):100-110.

Farias, A. N. S., M. Silva, J. B. S. Oliveira, L. B. Rocha \& K. R. Santos. 2013. Diagnóstico de parasitos gastrointestinais em cães do município de Bom Jesus, Piauí. Rev. Acad. Ciências Agr. e Ambient.11(4):431-435.

Guimarães, A. M., E. G. L. Alves, G. F. Rezende \& M. C. Rodrigues. 2005. Ovos de Toxocara sp. e larvas de Ancylostoma sp. em praça pública de Lavras, MG. Rev. Saude Publica. 39(2):293-295.

Gupta, M. 2016. Bullous cutaneous larva migrans - A case report. J Dermat. Dermatologic Surg. 20(1):65-66.

Hare, A. Q. \& C. Franco-Paredes. 2014. Ocular Larva Migrans: A Severe Manifestation of an Unseen Epidemic. Curr. Trop. Med. Reports $1(1): 69-73$.

IBGE - Instituto Brasileiro de Geografia e Estatística. Cidades. 2017.

Júnior, A. L. F., K. B. S. Araújo \& V. S. Medeiros. 2015. Ocorrência de parasitas com potencial zoonótico em fezes de cães coletadas em vias públicas da cidade de Natal. Rev. Hum. Ser. 1(1):52-59.

Leite, L. C. 2013. Ocorrência de ovos de endoparasitas em amostras de fezes de cães (Canis familiaris, Linnaeus, 1758) coletadas em vias públicas da cidade de Guarapuava-ParanáBrasil. Ambiência. 9(3): 619-626.

Mascarini-Serra, L. M. , C. A. Telles, M. S. Prado, S. A. Mattos, A. Strina, N. M. Alcantara-Neves \& M. L. Barreto. 2010. Reductions in the prevalence and incidence of geohelminth infections following a city-wide sanitation program in a Brazilian Urban Centre. PLoS Negl Trop Dis. 4(2):e588.

Moro, F. C. B., J. B. Pradebon, H. T. Santos \& E. Querol. 2008. Ocorrência de Ancylostoma sp. e Toxocara sp. em praças e parques públicos dos municípios de Itaqui e Uruguaiana, fronteira oeste do Rio Grande do Sul. Bio. pampeana. 6(1): 25-29.

Mota, A. F., V. Machado, S. P. Viegas, A. Emílio \& M. Vicente. 2016. Toxocara canis, o passageiro clandestino de um voo... Nascer e Crescer. 2016;25(2): 113-117.

Novaes, M.T. \& I. F. V. Martins. 2015. Avaliação de diferentes técnicas parasitológicas no diagnóstico de helmintoses caninas. Rev. Bras. Med. Vet., 37(1):71-76.

Oliveira, C. B., A. S. Silva \& S. G. Monteiro. 2007. Ocorrência de parasitas em solos de praças infantis nas creches municipais de Santa Maria - RS, Brasil. Rev. da Fac. Zootec. Veterinária e Agron. 14(1):174-179.

Ribeiro, K. L., T. D. Freitas, M. C. Teixeira, F. A. P. Araújo \& L. B. L. F. Mardini. 2013. Avaliação da ocorrência de formas parasitárias no solo de praças públicas do município de Esteio (RS). Ciência Anim. 11(1): 59-64.

Rodrigues, A. A. M., R. S. Corrêa, F. S. Souza, R. S. Lisbôa \& R. O. Pessoa. 2014. Ocorrência de parasitos zoonóticos em fezes de caes em áreas públicas em duas diferentes comunidades na Reserva Desenvolvimento Sustentável do Tupé, Amazonas. Rev. Bras. Hig. e San. Anim. 8(3):138-146.

Santarem, V. A., L. L. C. Bin \& M. C. A. Silva. 2012. Contaminação do solo por ovos de Toxocara sp. em assentamento rural de Mirante do Paranapanema, São Paulo, Brasil. Sem. Cie. Agr. 33(4):1525-1530.

Santos, G. M., S. A. Silva, A. P. Barbosa \& D. M. B. Campos. 2009. Investigação soroepidemiológica sobre a larva migrans visceral por Toxocara canis em usuários de serviços de saúde de Goiânia - Goiás. Rev. Pat. Trop. 38(3): 197-206.

Sommerfelt, I. E., N. Cardillo, C. López, M. Ribicich, C. Gallo \& A. Franco. 2006. Prevalence of Toxocara cati and other parasites in cats' faeces collected from the open spaces of public institutions: Buenos Aires, Argentina. Vet. Parasitol. 140(3-4):296-301. 
Souza, F. D., T. L. Mamede-Nascimento \& C. S. Santos. 2007. Encontro de ovos e larvas de helmintos no solo de praças públicas na zona sul da cidade do Rio de Janeiro. Rev. Patol. Trop. 36(3): 247-253.

Souza, R. F., V. C. C. Dattoli, L. R. Mendonça, J. R. Jesus, T. Baqueiro T, C. C. Santana, N. M. Santos, S. M. Barrouin-Melo \& N. M. Alcantara-Neves. 2011. Prevalência e fatores de risco da infecção humana por Toxocara canis em Salvador, Estado da Bahia. Rev. Soc. Bras. Med. Trop. 44(4):516-519.

Tekely, E., B. Szostakiewicz, B. Wawrzycki, G. Kadziela-Wypyska, M. Juszkiewicz-Borowiec, A. Pietrzak \& G. Choorowska. 2013. Cutaneous larva migrans syndrome: a case report. Adv Dermat.y Allergol. 2(46): 119-21.

Willis, H. H. A. 1921. A Simple Levitation Method for the Detection of Hookworm Ova. Med. J. Aust. 8:375-376.

Recebido em 14.II.2018

Aceito em 01.VIII.2018 